九州大学学術情報リポジトリ

Kyushu University Institutional Repository

\title{
SOME REMARKS ON ESTIMATION OF DIFFUSION COEFFICIENTS FOR JUMP-DIFFUSIONS FROM FINITE SAMPLES
}

Shimizu, Yasutaka

Graduate School of Engineering Science, Osaka University

https://doi.org/10.5109/18993

出版情報: Bulletin of informatics and cybernetics. 40, pp.51-60，2008-12. Research Association of Statistical Sciences

バージョン :

権利関係 : 
SOME REMARKS ON ESTIMATION OF DIFFUSION COEFFICIENTS FOR JUMP-DIFFUSIONS FROM FINITE SAMPLES

by

Yasutaka SHIMIZU

Reprinted from the Bulletin of Informatics and Cybernetics Research Association of Statistical Sciences, Vol.40

FUKUOKA, JAPAN

2008 


\title{
SOME REMARKS ON ESTIMATION OF DIFFUSION COEFFICIENTS FOR JUMP-DIFFUSIONS FROM FINITE SAMPLES
}

\author{
By
}

\section{Yasutaka SнIмıZU*}

\begin{abstract}
Diffusion coefficients of jump-diffusion processes with finite Lévy measure are estimated from discrete observations at points $t_{i}^{n}=i / n(i=0,1, \ldots, n)$ using filtered conditional moments of increments. The estimation is based on the local time for jump-diffusions and the consistency result is obtained. This is an extension of the result for pure diffusion cases by Florens-Zmirou (1993).
\end{abstract}

Key Words and Phrases: diffusion coefficients, jump-diffusions, discrete observations, asymptotic filter, local times, infinitesimal moments.

\section{Introduction}

On a filtered probability space $\left(\Omega, \mathscr{F},\left(\mathscr{F}_{t}\right)_{0 \leq t \leq 1}, P\right)$, we consider an one-dimensional jump-diffusion process $X$ satisfying the following stochastic differential equation:

$$
X_{t}=X_{0}+\int_{0}^{t} a\left(X_{s-}\right) d s+\int_{0}^{t} b\left(X_{s-}\right) d W_{s}+\int_{0}^{t} \int_{\mathbb{R}} c\left(X_{s-}, z\right) \mu(d s, d z)
$$

where $X_{0}$ is a random variable, coefficients $a, b$ and $c$ are measurable functions such that $a: \mathbb{R} \rightarrow \mathbb{R}, b: \mathbb{R} \rightarrow \mathbb{R}$ and $c: \mathbb{R} \times \mathbb{R} \rightarrow \mathbb{R}$, respectively, $W$ is a Wiener process, $\mu(d t, d z)$ is a Poisson random measure with the Lévy density $\pi ; E[\mu(d t, d z)]=\pi(z) d z d t$. We assume throughout this paper that $\int_{\mathbb{R}} \pi(z) d z<\infty$. Therefore (1) is well defined.

This paper is devoted to the nonparametric estimation of the diffusion function $\beta(\cdot):=b^{2}(\cdot)$ from discrete observations obtained in $[0,1]$-time interval, that is, $\left\{X_{t_{i}^{n}}\right\}_{i=0}^{n}$, where $t_{i}^{n}=i / n ; i=0,1, \ldots, n$.

Recently, several authors have studied the asymptotic inference for discretely observed jump-diffusions; see e.g. Bandi and Nguyen (2003), Mancini (2004), Shimizu and Yoshida (2006) and Shimizu (2006a,b) etc. All their works are discussed under the sampling scheme such that $t_{i}^{n}=i \times h_{n} ; i=0,1, \ldots, n$ for a sequence $h_{n}>0$ satisfying $h_{n} \rightarrow 0$ and $n h_{n} \rightarrow \infty$ as $n \rightarrow \infty$. This is because they tried to estimate not only the diffusion but also the drift and the Lévy characteristics such as $a(x), c(x, z)$ and $\pi$. We know well that it is impossible to estimate these characteristics without the asymptotics $n h_{n} \rightarrow \infty$. However it is nothing unusual in applications that the terminal $n h_{n}$ is fixed. In this paper we suppose that the observations are obtained in [0,1]-interval,

\footnotetext{
* Graduate School of Engineering Science, Osaka University, 1-3, Machikaneyama-cho, Toyonaka-shi, Osaka, 560-8531 Japan. tel +81-6-6850-6487 yasutaka@sigmath.es.osaka-u.ac.jp
} 
and try to estimate the diffusion function with the estimation of the drift and the Lévy characteristics abandoned.

When $X$ is a diffusion process without jumps; $\mu \equiv 0$, Florens-Zmirou (1993) proposed a nonparametric estimator of the diffusion coefficient in view of the fact that $t^{-1} E\left[\left(X_{s+t}-X_{s}\right)^{2} \mid X_{s}=x\right] \rightarrow \beta(x)$ as $t \rightarrow 0$ from the same type of observations as ours. However when $X$ is a jump-diffusion, the limit is not only $\beta(\cdot)$. Bandi and Nguyen (2003) extended their work to the case of jump-diffusions although the sampling scheme is different; $h_{n} \rightarrow 0$ and $n h_{n} \rightarrow \infty$. They also focused on the fact that

$$
\lim _{t \rightarrow 0} \frac{1}{t} E\left[\left(X_{s+t}-X_{s}\right)^{2} \mid X_{s}=x\right]=\beta(x)+\int_{\mathbb{R}} c^{2}(x, z) \pi(z) d z,
$$

and discretized the above conditional expectation when $t$ is small by using the local time estimators for $X$. Such a procedure is essentially the same as in Florens-Zmirou (1993), but the assumption that $n h_{n} \rightarrow \infty$ is necessary since the right-hand side of (2) includes the Lévy characteristics. Moreover the fact (2) clearly does not indicate the possibility of separate estimation of $\beta(\cdot)$ and $\int c^{2}(\cdot, z) \pi(z) d z$.

Our goal in this paper is to estimate $\beta(\cdot)$ separately under the sampling scheme that $n h_{n}$ is fixed. In order to execute the separate estimation as above, we need to discriminate whether the increment of neighboring data $\Delta_{i} X^{n}=X_{t_{i}^{n}}-X_{t_{i-1}^{n}}$ includes a jump or does not. An asymptotic filter proposed by Shimizu and Yoshida (2006) or Shimizu (2006a) enables us to judge whether a jump occurred or not in $\left(t_{i-1}^{n}, t_{i}^{n}\right]$. Although it is a stochastic judgment, the error rate asymptotically decreases as $n \rightarrow \infty$; see Section 2. Using increments that are expected not to include any jump, we take the similar argument to the one in Florens-Zmirou (1993); see Section 3, where some estimators of the local time of $X$ are presented. The estimator of $\beta(x)$; say $\hat{\beta}_{n}(x)$ is constructed in combination with their local time estimators. Our main result is for the consistency: $\lim _{n \rightarrow \infty} \hat{\beta}_{n}(x)=\beta(x)$ in probability for appropriate points $x \in \mathbb{R}$, which is presented in Theorem 3.7 .

\section{The basic idea of estimation}

Let $Y$ be an one-dimensional diffusion process that follows $d Y_{t}=\tilde{a}\left(Y_{t}\right) d t+\tilde{b}\left(Y_{t}\right) d W_{t}$. As already described, Florens-Zmirou (1993) focused attention to the following infinitesimal conditional moment to construct an estimator of $\tilde{b}^{2}$ :

$$
\lim _{\tau \rightarrow 0} \frac{1}{\tau} E\left[\left(Y_{t+\tau}-Y_{t}\right)^{2} \mid Y_{t}=x\right]=\tilde{b}^{2}(x) .
$$

This fact also indicates the possibility that we can extract only the diffusion part if we can identify the increment from the time $t$ to the first jump time. For that purpose, we introduce the asymptotic filter proposed in Shimizu (2006a), which is originally due to Shimizu and Yoshida (2006). The filter can separate the jumps of the process and the increment by diffusions asymptotically, and it helps us to discretize the expectation in (3). This is the basic idea in this paper. Indeed, the estimator of $\beta(\cdot)$ we propose in Theorem 3.7 later would look like a discrete approximation of $\tau^{-1} E\left[\left(Y_{t+\tau}-Y_{t}\right)^{2} \mid Y_{t}=x\right]$ for small $\tau$.

Before introducing the filter, we make the following assumptions, which is the similar setting as in Florens-Zmirou (1993) and Shimizu (2006a). 
A 1 For a constant $L$ and a function $\zeta(z)$ of polynomial growth in $z,|a(x)-a(y)|+$ $|b(x)-b(y)| \leq L|x-y|,|c(x, z)-c(y, z)| \leq \zeta(z)|x-y|$, and $|c(x, z)| \leq \zeta(z)(1+|x|)$.

A 2 The Lévy density $\pi$ is bounded and satisfies that $\int_{\mathbb{R}}|z|^{p} \pi(z) d z<\infty$ for any $p>0$. Moreover $\sup _{t \in[0,1]} E\left|X_{t}\right|^{p}<\infty$ for any $p>0$.

A 3 There exists $c_{0}>0$ such that $\inf _{x \in \mathbb{R}}|c(x, z)| \geq c_{0}|z|$ in a neighborhood of $z=0$.

A 4 The coefficient a is bounded and twice continuously differentiable with bounded derivatives. The coefficient $b$ is three times continuously differentiable with bounded derivatives such that there exists constants $k$ and $K$ with $0<k \leq b(x) \leq K$.

In the sequel, we use the following notation. Denote by $\Delta_{i} X^{n}:=X_{t_{i}^{n}}-X_{t_{i-1}^{n}}$; the increments of $X$ in the interval $\left(t_{i-1}^{n}, t_{i}^{n}\right]$, and denote by $J_{i}^{n}$ the number of jumps in that interval. The symbol $P_{i-1}^{n}$ stands for the conditional probability on $\sigma$-field $\mathscr{F}_{t_{i-1}^{n}}$, and $E_{i-1}^{n}$ means that the expectation with respect to $P_{i-1}^{n}$, that is, $P_{i-1}^{n}\{\cdot\}=P\left\{\cdot \mid \mathscr{F}_{t_{i-1}^{n}}\right\}$ and $E_{i-1}^{n}[\cdot]=E\left[\cdot \mid \mathscr{F}_{t_{i-1}^{n}}\right]$. Moreover $X \lesssim Y$ means there exists a constant $c>0$ such that $X \leq c Y$. Let $u_{n}$ be a real valued sequence, and $R: \mathbb{R} \times \mathbb{R} \rightarrow \mathbb{R}$ be a function for which there exists a constant $C$ such that $R\left(u_{n}, x\right) \lesssim u_{n}(1+|x|)^{C}$ for all $x \in \mathbb{R}^{d}$ and $n \in \mathbb{N}$.

The following lemma is due to Shimizu (2006a), which is the key result in this paper.

Lemma 2.1. Suppose Assumptions $A$ 1-A 3. Fix an arbitrary constant $L>0$ and $\rho \in[0,1 / 2)$. Then the following inequalities are valid for any $p \geq 1$ and sufficiently large $n \in \mathbb{N}$ :

$$
\begin{aligned}
P_{i-1}^{n}\left\{\left|\Delta_{i} X^{n}\right| \leq L n^{-\rho}, J_{i}^{n}=0\right\} & =R\left(1, X_{t_{i-1}^{n}}\right), \\
P_{i-1}^{n}\left\{\left|\Delta_{i} X^{n}\right| \leq L n^{-\rho}, J_{i}^{n}=1\right\} & =R\left(n^{-(1+\rho)}, X_{t_{i-1}^{n}}\right), \\
P_{i-1}^{n}\left\{\left|\Delta_{i} X^{n}\right| \leq L n^{-\rho}, J_{i}^{n} \geq 2\right\} & =R\left(n^{-2}, X_{t_{i-1}^{n}}\right), \\
P_{i-1}^{n}\left\{\left|\Delta_{i} X^{n}\right|>L n^{-\rho}, J_{i}^{n}=0\right\} & =R\left(n^{-p}, X_{t_{i-1}^{n}}\right), \\
P_{i-1}^{n}\left\{\left|\Delta_{i} X^{n}\right|>L n^{-\rho}, J_{i}^{n}=1\right\} & =R\left(n^{-1}, X_{t_{i-1}^{n}}\right), \\
P_{i-1}^{n}\left\{\left|\Delta_{i} X^{n}\right|>L n^{-\rho}, J_{i}^{n} \geq 2\right\} & =R\left(n^{-2}, X_{t_{i-1}^{n}}\right) .
\end{aligned}
$$

This lemma implies that, under $n \rightarrow \infty$, we can judge that the interval $\left(t_{i-1}^{n}, t_{i}^{n}\right]$ has no jump if $\left|\Delta_{i} X^{n}\right| \leq L n^{-\rho}$ and the interval has a single jump if $\left|\Delta_{i} X^{n}\right|>L n^{-\rho}$, and that we can ignore the event which includes more than two jumps in that interval. Although this lemma holds for any $L>0$ and $\rho \in[0,1 / 2)$ if we take sufficiently large $n \in \mathbb{N}$, it is important in applications to choose $L$ and $\rho$ suitably for fixed $n$. On some methods to choose a suitable $(L, \rho)$, see Shimizu (2007b).

Let us define the further notation: $\mathcal{C}_{i}^{n}=\cup_{j=1}^{2} \mathcal{C}_{i, j}^{n} ; i=1, \ldots, n$, where

$$
\begin{aligned}
& \mathcal{C}_{i, j}^{n}:=\left\{\left|\Delta_{i} X^{n}\right| \leq L n^{-\rho}, J_{i}^{n}=j\right\} \quad(j=0,1), \\
& \mathcal{C}_{i, 2}^{n}:=\left\{\left|\Delta_{i} X^{n}\right| \leq L n^{-\rho}, J_{i}^{n} \geq 2\right\} .
\end{aligned}
$$

Thanks to the previous lemma, we obtain the following estimates for moments of the increments $\Delta_{i} X^{n}$, which are provided in Shimizu and Yoshida (2006), Proposition 3.2. Therefore their proofs are omitted here. 
Lemma 2.2. Suppose Assumptions A 1-A 3. Then

$$
\begin{aligned}
E_{i-1}^{n}\left[\Delta_{i} X^{n} \mathbf{1}\left(\mathcal{C}_{i, 0}^{n}\right)\right] & =a\left(X_{t_{i-1}^{n}}\right) n^{-1}+R\left(n^{-2}, X_{t_{i-1}^{n}}\right), \\
E_{i-1}^{n}\left[\left(\Delta_{i} X^{n}\right)^{2} \mathbf{1}\left(\mathcal{C}_{i, 0}^{n}\right)\right] & =\beta\left(X_{t_{i-1}^{n}}\right) n^{-1}+R\left(n^{-2}, X_{t_{i-1}^{n}}\right), \\
E_{i-1}^{n}\left[\left(\Delta_{i} X^{n}\right)^{3} \mathbf{1}\left(\mathcal{C}_{i, 0}^{n}\right)\right] & =R\left(n^{-2}, X_{t_{i-1}^{n}}\right), \\
E_{i-1}^{n}\left[\left(\Delta_{i} X^{n}\right)^{4} \mathbf{1}\left(\mathcal{C}_{i, 0}^{n}\right)\right] & =3 \beta^{2}\left(X_{t_{i-1}^{n}}\right) n^{-2}+R\left(n^{-3}, X_{t_{i-1}^{n}}\right),
\end{aligned}
$$

where $\mathbf{1}(A)$ is the indicator function of a set $A$.

REMARK. Under the same assumptions as in Lemma 2.2, we see that

$$
E_{i-1}^{n}\left[\left|\Delta_{i} X^{n}\right|^{N} \mathbf{1}\left(\mathcal{C}_{i, 0}^{n}\right)\right]=R\left(n^{-N / 2}, X_{t_{i-1}^{n}}\right)
$$

for any $N \geq 1$. See Remark 3.1 in Shimizu and Yoshida (2006).

In the next section, we propose some estimators of the local time of $X$, which will play the role of $\tau$ in (3), and together with Lemma 2.2 enables us to discretize the infinitesimal conditional moments in (3).

\section{Local time estimates}

Before the discussion for estimators of the local time, we shall state some auxiliary results for the local time of semimartingales.

Notice that the assumption $\int_{\mathbb{R}} \pi(z) d z<\infty$ implies that $\sum_{0 \leq t \leq 1}\left|\Delta X_{t}\right|<\infty$ a.s., which is an important hypothesis for the following useful lemma; see e.g. Protter (2004).

LEMMA 3.1. We denote by $L_{t}(x)$ the local time of the jump-diffusion (1) in $x$ during $[0, t]$, then $L_{t}(x)$ has a version with càdlàg in $x$ and continuous in $t$, and the following equalities hold true:

$$
\begin{aligned}
L_{t}(x) & =L_{t}(x+)=\lim _{\epsilon \rightarrow 0} \frac{1}{\epsilon} \int_{0}^{t} \mathbf{1}\left(\left\{x \leq X_{s} \leq x+\epsilon\right\}\right) d[X, X]_{s}^{c} \quad \text { a.s. }, \\
L_{t}(x-) & =\lim _{\epsilon \rightarrow 0} \frac{1}{\epsilon} \int_{0}^{t} \mathbf{1}\left(\left\{x-\epsilon \leq X_{s} \leq x\right\}\right) d[X, X]_{s}^{c} \quad \text { a.s. }
\end{aligned}
$$

where $[X, X]^{c}$ is the path-by-path continuous part of the quadratic variation of $X$.

Moreover we denote by $\bar{L}_{t}(x)$ the symmetric version of the local time, that is,

$$
\bar{L}_{t}(x):=\frac{L_{t}(x+)+L_{t}(x-)}{2}=\lim _{\epsilon \rightarrow 0} \frac{1}{2 \epsilon} \int_{0}^{t} \mathbf{1}\left(\left\{\left|X_{s}-x\right| \leq \epsilon\right\}\right) d[X, X]_{s}^{c} \quad \text { a.s. }
$$

The next lemma is known as the occupation time formula.

LEMma 3.2. Let $X$ be a semimartingale with the local time $L_{t}(x)$ in $x$ during $[0, t]$, and let $g$ be a bounded Borel function. Then

$$
\int_{-\infty}^{\infty} L_{t}(x) g(x) d x=\int_{0}^{t} g\left(X_{s-}\right) d[X, X]_{s}^{c} \quad \text { a.s. }
$$


Now, let us construct estimators of the local time $\bar{L}_{t}(\cdot)$ from the data $\left(X_{t_{i}^{n}}\right)_{0 \leq i \leq n}$ using the asymptotic filter $\mathcal{C}^{n}:=\left\{\mathcal{C}_{i}^{n}\right\}_{i=1}^{n}$, and show their asymptotic behaviors.

First we prepare a lemma. Hereafter we use a convention $\sum_{i=1}^{0} \equiv 0$ and use

$$
I^{+}(y)=\mathbf{1}(\{0 \leq y \leq 1\}), \quad I^{-}(y)=\mathbf{1}(\{-1 \leq y \leq 0\}), \quad I(y)=\frac{1}{2} \mathbf{1}(\{|y| \leq 1\}) .
$$

Lemma 3.3. Suppose Assumption A 4. Let $\left\{\delta_{n}\right\}_{n \in \mathbb{N}}$ be a sequence such that $\delta_{n} \rightarrow 0$ and $n \delta_{n}{ }^{2} \rightarrow \infty$ as $n \rightarrow \infty$, and let $\Lambda_{n}=\sqrt{n} e^{-C n \delta_{n}{ }^{2}}$ for a constant $C>0$. Then it follows for each $i=1, \ldots, n$ and any $s \in\left(t_{i-1}^{n}, t_{i}^{n}\right]$ that

$$
\left\|\left\{I\left(\frac{X_{t_{i-1}^{n}}}{\delta_{n}}\right)-I\left(\frac{X_{s-}}{\delta_{n}}\right)\right\} \mathbf{1}\left(\left\{J_{i}^{n}=0\right\}\right)\right\|_{L^{1}}=O\left(\Lambda_{n}\right)
$$

as $n \rightarrow \infty$.

Proof. Let $Y$ be a strong solution process to the SDE $d Y_{t}=a\left(Y_{t}\right) d t+b\left(Y_{t}\right) d W_{t}$ and $Y_{t_{i-1}^{n}}=X_{t_{i-1}^{n}}$. Then $\left(X_{t}\right)_{t \in\left(t_{i-1}^{n}, t_{i}^{n}\right]}$ is indistinguishable from $\left(Y_{t}\right)_{t \in\left(t_{i-1}^{n}, t_{i}^{n}\right]}$ on the set $\left\{J_{i}^{n}=0\right\}$. Hence it follows for any $s \in\left(t_{i-1}^{n}, t_{i}^{n}\right] ; i=1, \ldots, n$, that

$$
\begin{gathered}
\left\|\left\{I\left(\frac{X_{t_{i-1}^{n}}}{\delta_{n}}\right)-I\left(\frac{X_{s-}}{\delta_{n}}\right)\right\} \mathbf{1}\left(\left\{J_{i}^{n}=0\right\}\right)\right\|_{L^{1}} \\
\quad \leq\left\|\mathbf{1}\left(\left\{\left|X_{t_{i-1}^{n}}\right| \leq \delta_{n}\right\}\right)-\mathbf{1}\left(\left\{\left|Y_{s-}\right| \leq \delta_{n}\right\}\right)\right\|_{L^{1}} \\
\quad=\left\|\mathbf{1}\left(\left\{\left|X_{t_{i-1}^{n}}\right| \leq \delta_{n},\left|Y_{s-}\right|>\delta_{n}\right\}\right)\right\|_{L^{1}}+\left\|\mathbf{1}\left(\left\{\left|X_{t_{i-1}^{n}}\right|>\delta_{n},\left|Y_{s-}\right| \leq \delta_{n}\right\}\right)\right\|_{L^{1}} \\
\quad=P\left\{\left|X_{t_{i-1}^{n}}\right| \leq \delta_{n},\left|Y_{s-}\right|>\delta_{n}\right\}+P\left\{\left|X_{t_{i-1}^{n}}\right|>\delta_{n},\left|Y_{s-}\right| \leq \delta_{n}\right\} .
\end{gathered}
$$

Here we denote by $p_{t}(x, y)$ the transition kernel of the process $Y$ from $x$ to $y$ in time $t$. Then it follows under A 4 that

$$
p_{t}(x, y) \lesssim \frac{1}{\sqrt{t}} \exp \left(-\frac{c(x-y)^{2}}{2 t}\right)
$$

for a positive constant $c$; see Friedman (1964) p257. Therefore we easily see that $\sup _{|x|<\delta_{n}}\left|p_{t}(x, y)\right| \lesssim t^{-1 / 2} \exp \left(-c y^{2} / 2 t\right)$; see also Lemma 1 in Florens-Zmirou (1993). Therefore, denoting by $D_{i}^{n}(d x)$ the distribution of $X_{t_{i-1}^{n}}$, we have

$$
\begin{aligned}
P\left\{\left|X_{t_{i-1}^{n}}\right| \leq \delta_{n},\left|Y_{s-}\right|>\delta_{n}\right\} & =\int_{-\delta_{n}}^{\delta_{n}} \int_{|y|>\delta_{n}} p_{s-t_{i-1}^{n}}(x, y) d y D_{i}^{n}(d x) \\
& \lesssim \int_{-\delta_{n}}^{\delta_{n}} \int_{|y|>\delta_{n}} \frac{1}{\sqrt{s-t_{i-1}^{n}}} e^{-\frac{c y^{2}}{2\left(s-t_{i-1}^{n}\right)}} d y D_{i}^{n}(d x) \\
& \lesssim \sqrt{n} e^{-C n \delta_{n}{ }^{2}} \quad(C>0) .
\end{aligned}
$$

The same argument as above is possible on the term $P\left\{\left|X_{t_{i-1}^{n}}\right|>\delta_{n},\left|Y_{s-}\right| \leq \delta_{n}\right\}$ since we also see by (9) that $\sup _{|y|<\delta_{n}}\left|p_{t}(x, y)\right| \lesssim t^{-1 / 2} \exp \left(-c^{\prime} y^{2} / 2 t\right) \quad\left(c^{\prime}>0\right)$. 
Lemma 3.4. Suppose Assumptions $A$ 1-A 4, and that $\delta_{n} \downarrow 0, n \delta_{n}{ }^{2} \rightarrow \infty$ and $\delta_{n}{ }^{-1} \Lambda_{n} \rightarrow 0$ as $n \rightarrow \infty$. Let $g$ be a function that is bounded and Lipschitz continuous, and let

$$
G_{t}^{(n)}(x)=\frac{1}{n \delta_{n}} \sum_{i=1}^{[n t]} g\left(X_{t_{i-1}^{n}}\right) I\left(\frac{X_{t_{i-1}^{n}}-x}{\delta_{n}}\right) \mathbf{1}\left(\mathcal{C}_{i}^{n}\right)
$$

for an arbitrary $t \in(0,1]$. Then the following convergence holds true for any $x \in \mathbb{R}$ :

$$
\lim _{n \rightarrow \infty}\left\|G_{t}^{(n)}(x)-g(x) \beta^{-1}(x) \bar{L}_{t}(x)\right\|_{L^{1}}=0 .
$$

Proof. Notice the following decomposition:

$$
\begin{aligned}
& \| G_{t}^{(n)}(x)-g(x) \beta^{-1}(x) \bar{L}_{t}(x) \|_{L^{1}} \\
& \leq\left\|\frac{1}{n \delta_{n}} \sum_{i=1}^{[n t]} g\left(X_{t_{i-1}^{n}}\right) I\left(\frac{X_{t_{i-1}^{n}}-x}{\delta_{n}}\right) \mathbf{1}\left(\left\{J_{i}^{n}=0\right\}\right)-g(x) \beta^{-1}(x) \bar{L}_{t}(x)\right\|_{L^{1}} \\
&+\left\|\frac{1}{n \delta_{n}} \sum_{i=1}^{[n t]} g\left(X_{t_{i-1}^{n}}\right) I\left(\frac{X_{t_{i-1}^{n}}-x}{\delta_{n}}\right) \mathbf{1}\left(\mathcal{C}_{i, 1}^{n} \cup \mathcal{C}_{i, 2}^{n}\right)\right\|_{L^{1}} \\
&+\left\|\frac{1}{n \delta_{n}} \sum_{i=1}^{[n t]} g\left(X_{t_{i-1}^{n}}\right) I\left(\frac{X_{t_{i-1}^{n}}-x}{\delta_{n}}\right) \mathbf{1}\left(\left\{\left|\Delta_{i} X^{n}\right|>L n^{-\rho}, J_{i}^{n}=0\right\}\right)\right\|_{L^{1}} \\
&=: \quad \alpha_{1}+\alpha_{2}+\alpha_{3} .
\end{aligned}
$$

On the second term $\alpha_{2}$, it is easily seen that $\alpha_{2} \leq \delta_{n}{ }^{-1} P\left\{\mathcal{C}_{i, 1}^{n} \cup \mathcal{C}_{i, 2}^{n}\right\}$ since $g$ and $I$ are bounded. Thus $\alpha_{2}=O\left(\delta_{n}{ }^{-1} n^{-(1+\rho)}\right) \rightarrow 0$ as $n \rightarrow \infty$. Similarly, we see that $\alpha_{3} \rightarrow 0$.

Let us estimate the first term $\alpha_{1}$. In the sequel, we assume $t=1$ and $x=0$ without loss of generality; see Florens-Zmirou (1993), and denote by $G_{1}^{(n)}(0)=G^{(n)}$ and $\bar{L}_{1}(0)=\bar{L}$. We show $G^{(n)}$ converges to $g(0) \beta^{-1}(0) \bar{L}$ in $L^{1}$-sense.

Notice that

$$
\begin{aligned}
\alpha_{1} \leq & \left\|G^{(n)} \mathbf{1}\left(\left\{J_{i}^{n}=0\right\}\right)-\int_{0}^{1} \frac{g\left(X_{s-}\right)}{\delta_{n} \beta\left(X_{s-}\right)} I\left(\frac{X_{s-}}{\delta_{n}}\right) d[X, X]_{s}^{c}\right\|_{L^{1}} \\
& +\left\|\int_{0}^{1} \frac{g\left(X_{s-}\right)}{\delta_{n} \beta\left(X_{s-}\right)} I\left(\frac{X_{s-}}{\delta_{n}}\right) d[X, X]_{s}^{c}-g(0) \beta^{-1}(0) \bar{L}\right\|_{L^{1}} \\
=: & \gamma_{1}+\gamma_{2} .
\end{aligned}
$$

On the term $\gamma_{1}$, noticing that $d[X, X]_{t}^{c}=\beta\left(X_{t}\right) d t$, the Lipschitz condition for $g$, and 
that $g$ and $I$ are bounded function, it follows by Lemma 3.3 that

$$
\begin{aligned}
\gamma_{1} \leq & \frac{1}{\delta_{n}} \sum_{i=1}^{n} \int_{t_{i-1}^{n}}^{t_{i}^{n}}\left\|g\left(X_{t_{i-1}^{n}}\right)\left\{I\left(\frac{X_{t_{i-1}^{n}}}{\delta_{n}}\right)-I\left(\frac{X_{s-}}{\delta_{n}}\right)\right\} \mathbf{1}\left(\left\{J_{i}^{n}=0\right\}\right)\right\|_{L^{1}} d s \\
& +\frac{1}{\delta_{n}} \sum_{i=1}^{n} \int_{t_{i-1}^{n}}^{t_{i}^{n}}\left\|I\left(\frac{X_{s-}}{\delta_{n}}\right)\left\{g\left(X_{t_{i-1}^{n}}\right)-g\left(X_{s-}\right)\right\}\right\|_{L^{1}} d s \\
\lesssim & \delta_{n}{ }^{-1} \sum_{i=1}^{n} \int_{t_{i-1}^{n}}^{t_{i}^{n}}\left\|I\left(\frac{X_{t_{i-1}^{n}}}{\delta_{n}}\right)-I\left(\frac{X_{s-}}{\delta_{n}}\right)\right\|_{L^{1}} d s+\delta_{n}{ }^{-1} \sum_{i=1}^{n} \int_{t_{i-1}^{n}}^{t_{i}^{n}}\left\|X_{s-}-X_{t_{i-1}^{n}}\right\|_{L^{1} d s} \\
= & O\left(\delta_{n}^{-1}\left(\Lambda_{n}+n^{-1 / 2}\right)\right) \rightarrow 0 \quad(n \rightarrow \infty) .
\end{aligned}
$$

On the term $\gamma_{2}$, thanks to Lemma 3.2, we obtain that

$$
\begin{aligned}
\gamma_{2}= & \left\|\int_{-\infty}^{\infty} \bar{L}_{1}(r) \frac{g(r)}{\delta_{n} \beta(r)} I\left(\frac{r}{\delta_{n}}\right) d r-g(0) \beta^{-1}(0) \bar{L}\right\|_{L^{1}} \\
= & \int_{\mathbb{R}}\left\|\frac{g\left(\delta_{n} q\right)}{\beta\left(\delta_{n} q\right)} \bar{L}_{1}\left(\delta_{n} q\right)-\frac{g(0)}{\beta(0)} \bar{L}\right\|_{L^{1}} I(q) d q \\
\lesssim & \frac{1}{2} \int_{0}^{\infty}\left\|\bar{L}\left(\delta_{n} q\right)-\bar{L}_{1}(0+)\right\|_{L^{1}} I(q) d q \\
& +\frac{1}{2} \int_{-\infty}^{0}\left\|\bar{L}\left(\delta_{n} q\right)-\bar{L}_{1}(0-)\right\|_{L^{1}} I(q) d q \\
& +\|\bar{L}\|_{L^{1}} \int_{\mathbb{R}}\left|\frac{g\left(\delta_{n} q\right)}{\beta\left(\delta_{n} q\right)}-\frac{g(0)}{\beta(0)}\right| I(q) d q .
\end{aligned}
$$

Here we see that $\bar{L}_{1}\left(\delta_{n} q\right)$ is uniformly integrable since $\sup _{n, q:|q| \leq 1} E\left|\bar{L}\left(\delta_{n} q\right)\right|^{2}<\infty$, which can be shown by Assumption A 2 and Tanaka's formula (e.g. Protter (2004), p.213, Theorem 68). Moreover $\bar{L}\left( \pm \delta_{n} q\right) \rightarrow \bar{L}(0 \pm)$ a.s. by Lemma 3.1. Hence $\bar{L}\left( \pm \delta_{n} q\right) \rightarrow \bar{L}(0 \pm)$ in $L^{1}$. Furthermore the bounded term $g\left(\delta_{n} q\right) \beta^{-1}\left(\delta_{n} q\right) \rightarrow g(0) \beta^{-1}(0)$ by the continuity. Therefore the last three terms converge to zero as $n \rightarrow \infty$ by the Lebesgue convergence theorem since $I(q) d q$ is a probability measure. This completes the proof.

REMARK. Suppose the same assumptions as in Lemma 3.4, and let

$$
\tilde{G}_{t}^{(n)}(x)=\frac{1}{n \delta_{n}} \sum_{i=1}^{[n t]} g\left(X_{t_{i-1}^{n}}\right) I\left(\frac{X_{t_{i-1}^{n}}-x}{\delta_{n}}\right),
$$

Then we also see that $\lim _{n \rightarrow \infty}\left\|\tilde{G}_{t}^{(n)}(x)-g(x) \beta^{-1}(x) \bar{L}_{t}(x)\right\|_{L^{1}}=0$ for all $x \in \mathbb{R}$ since

$$
\begin{aligned}
\left\|G_{t}^{(n)}(x)-\tilde{G}_{t}^{(n)}(x)\right\|_{L^{1}} & =\left\|\frac{1}{n \delta_{n}} \sum_{i=1}^{[n t]} g\left(X_{t_{i-1}^{n}}\right) I\left(\frac{X_{t_{i-1}^{n}}-x}{\delta_{n}}\right) \mathbf{1}\left(\left\{\left|\Delta_{i} X^{n}\right|>L n^{-\rho}\right\}\right)\right\|_{L^{1}} \\
& =O\left(n^{-1} \delta_{n}^{-1}\right),
\end{aligned}
$$

which tends to zero as $n \rightarrow \infty$. 
By Remark 3., we have the following.

Corollary 3.5. Suppose the same assumptions as in Lemma 3.4. Then

$$
\begin{aligned}
L_{t}^{(n)}(x) & :=\frac{1}{n \delta_{n}} \sum_{i=1}^{[n t]} I\left(\frac{X_{t_{i-1}^{n}}-x}{\delta_{n}}\right) \rightarrow \beta^{-1}(x) \bar{L}_{t}(x), \\
B_{t}^{(n)}(x) & :=\frac{1}{n \delta_{n}} \sum_{i=1}^{[n t]} \beta\left(X_{t_{i-1}^{n}}\right) I\left(\frac{X_{t_{i-1}^{n}}-x}{\delta_{n}}\right) \rightarrow \bar{L}_{t}(x)
\end{aligned}
$$

in $L^{1}$-sense as $n \rightarrow \infty$.

The above estimator $B_{t}^{(n)}(\cdot)$ still includes the unknown function $\beta$. Therefore we provide its empirical version as follows.

LEMMA 3.6. Let

$$
\hat{B}_{t}^{(n)}(x)=\frac{1}{\delta_{n}} \sum_{i=1}^{[n t]} I\left(\frac{X_{t_{i-1}^{n}}-x}{\delta_{n}}\right)\left(\Delta_{i} X^{n}\right)^{2} \mathbf{1}\left(\mathcal{C}_{i}^{n}\right) .
$$

Suppose the same assumptions as in Lemma 3.4. Then, for all $x \in \mathbb{R}$ and $t \in(0,1]$

$$
\hat{B}_{t}^{(n)}(x) \stackrel{p}{\longrightarrow} \bar{L}_{t}(x)
$$

Proof. We shall show that $\hat{B}_{t}^{(n)}(x)-B_{t}^{(n)}(x) \stackrel{p}{\longrightarrow} 0$, then Corollary 3.5 yields the consequence. For that purpose, it suffices to show the following two conditions; see Genon-Catalot and Jacod (1993), Lemma 9: as $n \rightarrow \infty$,

$$
\begin{array}{r}
\sum_{i=1}^{[n t]} E_{i-1}^{n}\left[\frac{1}{\delta_{n}} I\left(\frac{X_{t_{i-1}^{n}}-x}{\delta_{n}}\right)\left(\Delta_{i} X^{n}\right)^{2} \mathbf{1}\left(\mathcal{C}_{i}^{n}\right)\right]-B_{t}^{(n)}(x) \stackrel{p}{\longrightarrow} 0 \\
\sum_{i=1}^{[n t]} E_{i-1}^{n}\left[\left|\frac{1}{\delta_{n}} I\left(\frac{X_{t_{i-1}^{n}}-x}{\delta_{n}}\right)\left(\Delta_{i} X^{n}\right)^{2} \mathbf{1}\left(\mathcal{C}_{i}^{n}\right)\right|^{2}\right] \stackrel{p}{\longrightarrow} 0 .
\end{array}
$$

On (11), we see by Lemma 2.2, (5) that

$$
\begin{aligned}
\sum_{i=1}^{[n t]} E_{i-1}^{n} & {\left[\frac{1}{\delta_{n}} I\left(\frac{X_{t_{i-1}^{n}}-x}{\delta_{n}}\right)\left(\Delta_{i} X^{n}\right)^{2} \mathbf{1}\left(\mathcal{C}_{i}^{n}\right)\right]-B_{t}^{(n)}(x) } \\
& =\frac{1}{n \delta_{n}} \sum_{i=1}^{[n t]} I\left(\frac{X_{t_{i-1}^{n}}-x}{\delta_{n}}\right) E_{i-1}^{n}\left[n\left(\Delta_{i} X^{n}\right)^{2} \mathbf{1}\left(\mathcal{C}_{i}^{n}\right)-\beta\left(X_{t_{i-1}^{n}}\right)\right] \\
& =O_{p}\left(\frac{1}{n \delta_{n}}\right) \stackrel{p}{\longrightarrow} 0 .
\end{aligned}
$$


Moreover, on (12), we see by Lemma 2.2, (7) that

$$
\begin{aligned}
\sum_{i=1}^{[n t]} E_{i-1}^{n} & {\left[\left|\frac{1}{\delta_{n}} I\left(\frac{X_{t_{i-1}^{n}}-x}{\delta_{n}}\right)\left(\Delta_{i} X^{n}\right)^{2} \mathbf{1}\left(\mathcal{C}_{i}^{n}\right)\right|^{2}\right] } \\
& =\frac{1}{\delta_{n}{ }^{2}} \sum_{i=1}^{[n t]} I\left(\frac{X_{t_{i-1}^{n}}-x}{\delta_{n}}\right) E_{i-1}^{n}\left[\left(\Delta_{i} X^{n}\right)^{4} \mathbf{1}\left(\mathcal{C}_{i}^{n}\right)\right] \\
& =O_{p}\left(\frac{1}{n \delta_{n}{ }^{2}}\right) \stackrel{p}{\longrightarrow} 0 .
\end{aligned}
$$

This ends the proof.

The next theorem is our main result, which is the direct consequence from Corollary 3.5 and Lemma 3.6.

Theorem 3.7. Suppose Assumptions A 1-A 4, and that $\delta_{n} \downarrow 0, n \delta_{n}{ }^{2} \rightarrow \infty$ and $\delta_{n}{ }^{-1} \Lambda_{n} \rightarrow 0$ as $n \rightarrow \infty$. Then the estimator $\hat{\beta}_{n}(x):=\hat{B}_{1}^{(n)}(x) / L_{1}^{(n)}(x)$ is consistent to $\beta(x)$ for all $x \in \mathbb{R}$ with $\bar{L}_{1}(x)>0$ :

$$
\hat{\beta}_{n}(x) \stackrel{p}{\longrightarrow} \beta(x)
$$

as $n \rightarrow \infty$.

Remark. Mancini (2004) also proposes the same type of the asymptotic filter, which can also be applied to our purpose. However, in that case, the order condition for $\delta_{n}$ would be changed.

REMARK. Under the asymptotics $h_{n} \rightarrow 0$ and $n h_{n} \rightarrow \infty$, it would also be possible to estimate $\beta(\cdot)$ and $\int c^{2}(\cdot, z) \pi(z) d z$ separately, and also $a(\cdot)$ by operating the asymptotic filter to kernel estimators by Bandi and Nguyen (2003). In particular, when $c(x, z)$ is known, Shimizu (2007a) proposes a nonparametric estimator of $\int G(\cdot, z) \pi(z) d z$ for any known function $G$.

\section{Acknowledgement}

The author would like to thank the anonymous referees for helpful comments and suggestions. He is also grateful to Professor Sadanori Konishi for his help.

\section{References}

Bandi, M. and Nguyen, H. (2003). On the functional estimation of jump-diffusion models, J. Econometrics 116, 211-229.

Florens-Zmirou, D. (1993). On estimating the diffusion coefficient from discrete observations, J. Appl. Prob. 30, 790-804.

Friedman, A. (1964). Partial differential equations of parabolic type, Prentice-Hall, Inc., Englewood Cliffs, N.J. 
Genon-Catalot, V. and Jacod, J. (1993). On the estimation of the diffusion coefficient for multidimensional diffusion process, Ann. Inst. Henri Poincaré Prob. Statist. 29, 119-151.

Gihman, I. and Skorohod, V. (1972). Stochastic Differential Equation, Springer-Verlag, Berlin.

Mancini, C. (2004). Estimation of the characteristics of the jumps of a general Poissondiffusion model, Scand. Actuarial J. no. 1, 42-52.

Protter, P. (2004). Stochastic Integration and Differential Equations, Springer-Verlag, New York, 2nd ed.

Shimizu, Y. (2006a). Density estimation of Lévy measure for discretely observed diffusion processes with jumps, J. Japan Statist. Soc. 36, no. 1, 37-62.

Shimizu, Y. (2006b). M-estimation for discretely observed ergodic diffusion processes with infinitely many jumps, Statist. Infer. Stochastic Proc. 9, no. 2, 179-225.

Shimizu, Y. (2007a). . Semiparametric estimation of Lévy characteristics of jumpdiffusion models from sampled data, Proceedings of the 9th Japan-China Symposium on Statistics, 265-270, Hokkaido University, Japan.

Shimizu, Y. (2007b). A practical inference for discretely observed jump-diffusions from finite samples. to appear in J. Japan Statist. Soc.

Shimizu, Y. and Yoshida, N. (2006). Estimation of parameters for diffusion processes with jumps from discrete observations, Statist. Infer. Stochastic Proc. 9, no. 3, 227277.

Received December 12, 2007

Revised March 6, 2008 